\title{
Les traces du traducteur dans le discours de presse
}

\section{Translator's traces in newspaper discourse}

\author{
Dorota Lajus \\ Université Mikołaj Kopernik, Toruń \\ dorotalajus@umk.pl
}

\begin{abstract}
The interlocutory dialogicality of press discourse manifests itself, among other things, in the use of common expressions that give the impression that "it is the language that speaks, not its users" (Bonhomme, 2012, p. 77). Euphemisms, for example, are used to raise difficult or polemic inducing issues, such as poverty, exclusion, war, politics, etc. Meanwhile, expressions that have become common in the source language, and are consistent with generally accepted norms, do not always remain transparent during the translation process. Depending on the translator's choices, the phrases used in the target text can have various effects, ranging from the erasure of sensitive topics, to their hyperbolization. The subject of the paper is the comparison of articles published in the French monthly Le Monde diplomatique and their translations, which appeared on the pages of the Polish edition of this periodical. Its aim is to reveal the traces of the translator's presence, visible through his or her ways of interpreting euphemistic phrases, or toning down harsh expressions.
\end{abstract}

Keywords: press discourse, euphemism, traces of the translator

\section{INTRODUCTION}

L'objet de ce travail est une recherche des traces du traducteur laissées au cours de son opération traduisante, à travers une lecture comparative des articles de presse originaux et de leur traduction en polonais. Il s'agit donc d'une lecture confrontative dans les termes de Balcerzan (2011, p. 110), laquelle met en évidence les différences multiples entre l'original et la traduction.

La notion de traces du traducteur est liée à celle du troisième code (Frawley, p. 1984) qui est créé lors de la confrontation du texte source et de la langue 
cible : « le code (ou la langue) qui évolue pendant la traduction, et dans lequel le texte cible est rédigé, serait unique. Il s'agit d'un compromis entre les normes ou structures de la langue source et ceux de la langue cible» (Baker, 1998, p. 2). Ce troisième code dépendrait notamment des normes descriptives de traduction, autrement dit des tendances observables chez les traducteurs à choisir certaines méthodes de traduction.

Si nous acceptons que toute structuration linguistique est influencée par les contraintes liées à l'environnement et à une époque donnée de l'utilisation de la langue, et ce à n'importe quel moment, alors nous devons admettre que la structuration d'un texte traduit diffère de celle d'un original. En d'autres mots, la nature et les pressions du processus traductionnel doivent laisser des traces dans la langue utilisée par les traducteurs (Baker, 1996, p. $176 ; 1998$, p. 2).

Les traces de traduction, dans ce sens, sont des écarts par rapport à la langue cible. C'est pourquoi on les recherche en comparant des textes originaux et des traductions dans une même langue et dans des domaines proches (Baker, 1998, p. 2).

D'autre part, nous nous inspirons du travail de T. Tomaszkiewicz (2016) qui analyse les manifestations de la présence du traducteur dans les textes spécialisés et, s'appuyant sur Dąmbska-Prokop (2000, p. 227), distingue deux types de traces du traducteur. Le premier embrasse «celles liées à une recherche consciente des équivalences » (Tomaszkiewicz, 2016, p. 98) que l'on peut relever en comparant le texte traduit au texte source, et qui «se rapportent à des éléments jugés comme non transférables directement ou intraduisibles » (Tomaszkiewicz, 2016, p. 101). La deuxième catégorie comporte les traces autonomes, perceptibles sans la nécessité de voir l'original, telles que les notes ou les préfaces.

Notre étude prend appui sur un corpus d'articles publiés dans le mensuel français Le Monde diplomatique et leurs traductions parues dans l'édition polonaise de ce périodique. L'enquête est limitée à une diachronie de cinq ans de publication, entre février 2013 et février 2018. Il s'agit d'un magazine renommé et ayant plusieurs dizaines d'éditions internationales. Les traductions polonaises sont effectuées par des traducteurs réputés et expérimentés, et l'importance accordée à leur travail est visible dans le fait que pour chaque article traduit, on indique le nom du traducteur, ce qui n'est pas pratiqué dans tous les titres de presse. De plus, la ligne éditoriale de ce mensuel d'information et d'opinion est engagée, en l'occurrence en faveur de la gauche, ce qui peut avoir de l'influence entre autres sur la langue employée, relevant notamment du politiquement correct qui « vise à ne pas offenser des groupes humains lésés, défavorisés, dits souvent minoritaires, même si quantitativement ils peuvent ne pas l'être » (López Diaz, 2017, p. 19).

La traduction des textes de presse possède cette spécificité que le traducteur devient en même temps journaliste : 
C'est manifestement lorsqu'il traduit la presse que le traducteur se rapproche le plus du journaliste. De même qu'un traducteur littéraire a forcément en lui un écrivain latent, un traducteur de presse générale ou spécialisée prend plaisir à adopter la qualité d'un style journalistique correspondant au média et au public pour lesquels il traduit. À lui de recréer un titre et un chapeau accrocheurs, d'expliciter un sigle ou une réalité culturelle inédite, en bref d'adapter son texte à son lecteur, comme l'a fait le journaliste lorsqu'il a écrit le texte original (Lavault-Olléon \& Sauron, 2009, p. 2).

Pour cette raison, la comparaison des articles originaux en français et leurs traductions fait surgir des divergences dans le péritexte. Ainsi, les titres, et encore plus souvent les surtitres et les chapeaux dans Le Monde diplomatique polonais ne sont pas des traductions de l'original, mais des créations des traducteurs et rédacteurs car ils constituent des entités autonomes et polyfonctionnelles à propos desquelles les stratégies des rédactions changent en fonction de la langue et de la culture d'édition $^{1}$. Toutefois, il est à noter que quand on analyse la forme finale d'un article traduit, il est difficile de savoir lesquelles des modifications sont apportées par le traducteur et lesquelles par les rédacteurs. En ce qui concerne le péritexte, le traducteur propose les titres et les surtitres de l'article traduit, mais la forme finale en est donnée par le secrétaire de rédaction ${ }^{2}$.

La confrontation des deux versions montre également que le traducteur et le rédacteur adaptent l'article au public cible ${ }^{3}$. Ainsi, nous relevons des omissions de certains fragments ou des explicitations d'informations implicites dans l'original. Ces dernières sont placées dans le corps du texte. Certaines sont mises entre parenthèses, par exemple : Quai d'Orsay (Ministerstwo Spraw Zagranicznych) ('Ministère des affaires étrangères') ; Bercy (Siedziba francuskiego ministerstwa gospodarki przyp. ttum.) ('Siège du ministère français de l'économie - note du traducteur') ; hotel Matignon (siedziba premiera Francji) ('hôtel Matignon (siège du premier ministre français)'), etc. D'autres ajouts explicatifs ${ }^{4}$ sont insérés dans le texte sous forme de phrases ou de développement de phrase, comme le montre l'exemple ci-dessous :

\footnotetext{
${ }^{1}$ Exemple de titres : Haro sur Schengen, Strefa Schengen nie dla uchodźców ('zone Schengen pas pour les réfugiés') (janvier 2016) ; En banlieue, autoentrepreneur faute de mieux, Wszyscy zostańmy przedsiębiorcami ('devenons tous des entrepreneurs') (mars 2017); Les vieux habits de l'homme neuf, Emmanuel Macron i urok technokraty ('Emmanuel Macron et le charme du technocrate') (mars 2017).

${ }^{2}$ D'après les informations fournies par A.P. Studziński (président de l'éditeur de la version polonaise du Monde diplomatique) dans une correspondance e-mail du 2 août 2018.

${ }^{3}$ D'après A.P. Studziński, les décisions concernant l'omission d'un fragment du texte ou l'insertion d'un ajout explicatif incombent au rédacteur.

${ }^{4}$ Nous comprenons ici ajout (adjonction) dans le sens développé par Guidère (2010) et Tomaszkiewicz (2016) : «L'ajout d'informations inexistantes sur l'original par le biais d'une explicitation ou d'une expansion, que ce soit dans le corps du texte, en note de bas de page ou encore dans le glossaire » (Guidère, 2010, p. 86).
} 
(1) En France, après l'expulsion en octobre dernier W październiku, po wydaleniu z Francji Leonardy de Leonarda Dibrani, cette lycéenne interpellée Dibrani, licealistki zatrzymanej podczas szkolnej lors d'une sortie scolaire, M. Jean-François wycieczki, przewodniczący Unii na rzecz Ruchu Copé, président de l'Union pour un mouvement Ludowego (UMP) Jean-François Copé wzywał do populaire (UMP), a préconisé la suppression zniesienia zasilku AME, przyznawanego obcode l'AME, sous-entendant qu'il en allait de krajowcom, którzy nie mają dokumentów ani la survie du système français de protection sociale.

(A. Spire, décembre 2013)

pracy - sugerując, że w ten sposób można ocalić francuski system ochrony socjalnej.

(trad. A. Dwulit)

La lecture des textes permet aussi de dégager des traces d'interventions du traducteur et du rédacteur face aux différences culturelles sous forme de techniques adaptatives telles que l'emploi des hypéronymes (le revenu de solidarité active (RSA) (fr.) - zasitek (allocation) (RSA) (pol.)), (énarque - technokrata ('technocrate')), des équivalents fonctionnels (BFMTV (fr.) - TVN24 Biznes $i$ Świat (pol.)) etc.

Toutefois, l'objectif de ce travail est d'observer les stratégies des auteurs de l'édition polonaise vis-à-vis des euphémismes de la version française, ainsi que les marques d'une prise de position envers les informations données.

\section{DIALOGISME INTERDISCURSIF ET EUPHÉMISMES DE PRESSE}

Le discours de presse se caractérise entre autres par une hétérogénéité énonciative qui se manifeste par l'importance des citations, la polyphonie des voix et un dialogisme interdiscursif (Moirand, 2007, p. 12). Il emploie notamment des expressions euphémiques qui se font l'écho d'une voix collective : "on euphémise sous la pression d'autrui et à l'intention d'autrui. [...] la voix de l'Autre est intimement présente lors de la production d'un euphémisme » (Bonhomme, 2005, p. 240). Le terme d'euphémisme est issu de la rhétorique, Dumarsais l'a classé parmi les tropes stylistiques et l'a défini comme procédé de substitution : «L'euphémisme est une figure par laquelle on déguise des idées désagréables, odieuses ou tristes sous des noms qui ne sont point les noms propres de ces idées : ils leur servent comme de voile, et ils en expriment en apparence de plus agréables, de moins choquantes, ou de plus honnêtes, selon le besoin » (Dumarsais, 1988, p. 158). Dans notre travail, nous comprenons l'euphémisme comme " un procédé d'atténuation discursive en face des réalités risquées » (Bonhomme, 2005, p. 240), c'est-à-dire choquantes ou pénibles. Comme le remarque López Diaz, l'euphémisation peut se réaliser de diverses manières :

L'euphémisme peut se manifester formellement à travers des tournures, phrases, sigles, emprunts, abréviations, déformations, suppressions, etc., qui du point de vue sémantique ont une valeur d'hypéronymes, de faux synonymes, d'expressions vagues et approximatives [...] (López Diaz, 2012, p. 193). 
D'un côté, l'euphémisme fait partie d'une socioculture car il est « un effet de discours souvent lié à l'ensemble de valeurs sociohistoriques d'une langue-culture : c'est l'usage social qui transforme cet emploi en règles, en véritables normes de politesse » (Seoane, 2016, p. 272). D'un autre côté, la préférence de certaines formulations découle des opinions, et la réflexion sur l'euphémisation touche également des questions axiologiques : «Lorsque [1]es énonciations recourent à l'euphémisme, s'esquissent la construction d'un ethos et de certaines tendances idéologiques (assumées ou non) » (Seoane, 2016, p. 273).

Par ailleurs, la figuralité de l'expression se prête à une négociation, elle dépend non seulement du contexte et du locuteur, mais aussi de sa réception par l'interlocuteur. Ainsi, le récepteur repère ou non une figure et l'interprète : « un euphémisme est réussi lorsqu'à travers ses stratégies de masquage et de routinisation, il exerce des effets modérateurs et dédramatisants sur le récepteur, cela dans le sens souhaité par l'énonciateur» (Bonhomme, 2012, p. 79). En revanche, un euphémisme peut connaître un échec lorsque le lecteur le repère, en fait « une interprétation critique » ou parvient à «mettre en lumière l'expression littérale masquée sous la figure » (Bonhomme, 2012, p. 83). D'après M. Bonhomme (2012, pp. 83-87), trois sortes de réactions du récepteur (ici le traducteur et le rédacteur) peuvent entraîner l'échec réceptif de l'euphémisme. Premièrement, par la distanciation qui peut se manifester graphiquement par l'emploi des guillemets, deuxièmement, par le démasquage lorsque le récepteur dévoile la figuralité d'une expression et enfin, par la stigmatisation, c'est à dire le traitement polémique d'une formulation.

\section{LA TRADUCTION D’EXPRESSIONS EUPHÉMIQUES}

Nous avons relevé dans les articles français des formulations atténuantes qui ne sont pas des productions discursives idiolectales mais des expressions routinières et collectives qui se sont sédimentées dans les structures lexicalisées de la langue. Toutefois, lors du processus de traduction, ces expressions, devenues banales dans la langue de départ car entrées dans la doxa d'une société, ne restent pas toujours transparentes. Les traducteurs repèrent-ils des euphémismes, emploient-ils les expressions polonaises équivalentes, explicitent-ils des informations édulcorées?

\subsection{Les euphémismes équivalents}

Dans le corpus français analysé, nous relevons des exemples de lexèmes, considérés par plusieurs linguistes comme substituts euphémiques de dénominations plus directes. C'est notamment le cas des expressions : opération et intervention qui, en contexte, se rapportent à des actes de guerre. D'après le Petit Robert de la langue française $(P R)$, le terme opérations désigne un « ensemble de mouvements, de manœuvres, de combats qui permet d'atteindre un objectif, d'assurer la défense d'une 
position, le succès d'une attaque $»$. Le dictionnaire note que cette acception du terme remonte à l'année 1701. Bonhomme, Torre et Horak remarquent que le terme ne fait pas qu'atténuer, mais il présente aussi les combats sous un jour favorable : « Loin de se limiter à un traitement détensif qui réduit la gravité de la guerre, l'euphémisme opérations met simultanément en œuvre un autre traitement d'ordre axiologique : la mélioration qui en neutralise les ravages » (Bonhomme, Torre \& Horak, 2012, p. 7). Il en est de même avec intervention, ce terme polysémique n'explicite pas le sème /+combat/. De plus, dans certaines occurrences, on observe l'ellipse de l'adjectif militaire, ce qui rend implicite le caractère guerrier de l'intervention ou de l'opération en question. Ces dénominations, critiquées par certains comme relevant de la langue de bois, se sont pourtant sédimentées dans le discours de presse. Elles trouvent d'ailleurs des correspondants aussi routinisés dans la langue polonaise. Ainsi, les traducteurs ont rendu ces expressions françaises avec des formules polonaises correspondantes : opérations militaires - operacje wojskowe, opération - operacja, intervention militaire - interwencja wojskowa, zaangażowanie militarne etc :

\begin{tabular}{|c|c|}
\hline $\begin{array}{l}\text { (2) Lorsqu'ils devront caractériser les opérations } \\
\text { militaires françaises du début du XXIe siècle, } \\
\text { peut-être les historiens parlerons-ils de « ho- } \\
\text { quets stratégiques », tant le mouvement d'en- } \\
\text { semble des dix dernières années apparaît syn- } \\
\text { copé . } \\
\text { (O. Zajec, février 2013) }\end{array}$ & $\begin{array}{l}\text { Jeśli w przyszłości historycy zechcą scharakte- } \\
\text { ryzować francuskie operacje wojskowe z począt- } \\
\text { ku XXI w, będą prawdopodobnie mówić o ,stra- } \\
\text { tegicznej czkawce” - tak bardzo rytm wydarzeń } \\
\text { ostatniej dekady wydają się cechować nieregu- } \\
\text { larność i nieprzewidywalnośc. } \\
\text { (L. Mazur) }\end{array}$ \\
\hline $\begin{array}{l}\text { (3) Avec l'intervention des gardiens de la révo- } \\
\text { lution en Syrie et en Irak, l'Iran a, pour la pre- } \\
\text { mière fois de son histoire moderne, remporté } \\
\text { une bataille militaire à l'extérieur de ses fron- } \\
\text { tières. } \\
\text { (B. Hourcade, février 2018) }\end{array}$ & $\begin{array}{l}\text { Wraz z interwencją Korpusu Strażników Rewo- } \\
\text { lucji Islamskiej w Syrii i Iraku po raz pierwszy } \\
\text { w swojej historii współczesnej Iran wygrał bitwę } \\
\text { na zewnętrznym polu walki. } \\
\text { (Z.M. Kowalewski) }\end{array}$ \\
\hline
\end{tabular}

Dans l'exemple suivant, l'auteur reprend le terme frapper (de frappe aérienne) employé par les instances institutionnelles. Toutefois, il marque sa distance par rapport à ce rituel euphémique avec des guillemets qui montrent qu'il s'agit du discours d'un autre. De plus, dans sa phrase, il préfère employer un terme plus concret, bombarder. Le traducteur polonais le rend littéralement et garde les guillemets.

\begin{tabular}{|l|l|}
\hline (4) Les djihadistes ont calculé que les « croisés » & Dżihadyści wykalkulowali, że „krzyżowcy” i „bał- \\
et les « idolâtres » pouvaient bien bombarder & wochwalcy” mogliby uderzyć, tzn. bombardo- \\
(frapper) des villes syriennes, quadriller des & wać miasta syryjskie, patrolować prowincje irac- \\
provinces irakiennes, mais qu'ils ne parvien- & kie, ale nigdy nie byliby w stanie okupować ziemi \\
draient jamais à occuper durablement une terre & arabskiej. \\
arabe. & (Z.M. Kowalewski) \\
(S. Halimi, décembre 2015) & \\
\hline
\end{tabular}


Dans ces exemples, la comparaison des deux versions ne fait pas voir les traces d'une distanciation du traducteur par rapport à l'original.

\subsection{Le démasquage de l'euphémisme}

L'analyse du corpus fait relever des cas où les expressions atténuantes sont repérées par le traducteur, et où la réalité tabouisée est rendue explicite dans la version polonaise.

3.2.1. Malgré l'existence d'expressions équivalentes en polonais, certaines formules euphémisantes sont rendues par des expressions non-euphémiques. Ainsi, le verbe disparaître pour mourir est devenu un euphémisme conventionnel et de ce fait, parfaitement compréhensible. Le traducteur polonais traduit l'information explicitement sans chercher à la rendre par un euphémisme stéréotypé polonais :

\begin{tabular}{|l|l|}
\hline (5) Des intellectuels la promeuvent, certains expli- & Promują ją otwarcie niektórzy intelektualiści, jak \\
citement, comme la journaliste italienne Oria- & włoska dziennikarka Oriana Fallaci (zmarla \\
na Fallaci (disparue en 2006), citée dès la pre- & w 2006 r.), cytowana już w pierwszym zdaniu \\
mière ligne du premier chapitre d'Eurabia, & pierwszego rozdziału Eurabii, niemiecki ekono- \\
l'économiste allemand Thilo Sarrazin ou le ro- & mista Thilo Sarazzin czy francuski powieściopi- \\
mancier français Renaud Camus. & sarz Renaud Camus. \\
(R. Liogier, mai 2014) & (E. Cylwik)
\end{tabular}

De même, dans la citation suivante, on observe une ellipse de l'adjectif qualificatif militaire alors que le traducteur rend explicite le caractère sous-entendu de l'engagement :

\begin{tabular}{|l|l|}
\hline (6) Revendiquées par l'Organisation de l'Etat isla- & Popełnione 13 listopada br. w Paryżu mordy, do \\
mique (OEI), les tueries du 13 novembre der- & których przyznała się Organizacja Państwa Islam- \\
nier à Paris ont entraîné l'intensification de & skiego, pociągnęły za sobą wzmożone zaanga- \\
l'engagement occidental au Proche-Orient. & $\begin{array}{l}\text { żowanie wojskowe. } \\
\text { (S. Halimi, décembre 2015) }\end{array}$ \\
\hline
\end{tabular}

Dans le fragment ci-dessous, l'auteur, en parlant de la guerre au Yemen, reprend le terme dommages collatéraux, calque de l'anglais (collateral damage). Ce terme est enregistré par le $P R$ comme euphémisme de «conséquences secondaires non maîtrisées d'une opération militaire, notamment pertes civiles ». L'auteur de l'article marque avec des guillemets sa distance par rapport à cette formule utilisée dans les communiqués officiels. Le traducteur polonais ne remplace pas cette expression par le calque employé dans les médias polonais straty uboczne mais crée une formula- 
tion qui nomme explicitement les personnes touchées, en accentuant ainsi l'inappropriation de l'expression : ofiary uboczne ('victimes collatérales') :

(7) Les drones américains censés éliminer la menace djihadiste s'avèrent à la fois contre-productifs, en contribuant à légitimer les djihadistes aux yeux d'une population victime de dommages collatéraux, et symptomatiques d'un intérêt bien limité pour le pays - car ces armes caractérisent une «non-politique» et constituent un interventionnisme par défaut. (L. Bonnefoy, décembre 2017)
Amerykańskie drony, które miały wyeliminować dżihadystyczne zagrożenie, okazały się kontrproduktywne, przyczyniając się do usprawiedliwienia działań dżihadystów w oczach miejscowych ludzi, którzy często stają się ofiarami ubocznymi, a zarazem symptomatyczne dla bardzo ograniczonego zainteresowania krajem - ponieważ ten rodzaj broni jest typowy dla „depolityzacji” i ,biedainterwencjonizmu". (E. Cylwik)

3.2.2. Dans notre corpus nous retrouvons des expressions françaises employées fréquemment dans le discours public et pour lesquelles on ne trouve pas immédiatement d'équivalent polonais, soit que le référent soit spécifiquement français, soit que les expressions polonaises n'aient pas la même charge affective. Par exemple, dans le corpus français, nous avons relevé plusieurs fragments où l'on mentionne le problème de la pauvreté sans le nommer directement afin de ne pas dévaloriser ou discriminer les personnes concernées, et par là, choquer les lecteurs. Ces expressions peuvent être considérées comme un exemple du procédé observé déjà par Dumarsais, à savoir des « euphémismes de civilité du français quotidien qui épargnent l'humiliation en passant sous silence les inégalités de condition et de fortune » (Dumarsais, 1988, p. 158). Il est intéressant de remarquer que les traducteurs polonais déchiffrent ces expressions et les rendent avec des formulations plus directes, parfois même de manière hyperbolique.

Ainsi, les populations précaires, un terme de sciences sociales, employé communément pour parler des personnes pauvres ou touchées par de nouvelles formes de pauvreté (Pierret, 2013, p. 307) est rendu en polonais avec une dénomination qui expose plus directement les effets de la précarité : osoby najbiedniejsze ('les personnes les plus pauvres'). De la même façon, les plus précaires deviennent ludzie w najgorszej sytuacji ('les gens dans la pire situation') :

\footnotetext{
(8) Pour les classes moyennes du public, cette remise en question systématique de leur statut cadre mal avec le sentiment - erroné - que la puissance publique continue à dépenser toujours plus pour protéger les étrangers et les populations précaires.

(A. Spire, décembre 2013)

Ciągłe obniżanie się statusu przedstawicieli klas średnich pracujących $\mathrm{w}$ sferze publicznej zbiega się tu z ich - niesłusznym - wrażeniem, że władze wydają coraz więcej na pomoc obcokrajowcom i osobom najbiedniejszym.

(A. Dwulit)
} 
(9) Appartenant aux fractions inférieures du salariat, ils adhèrent d'autant plus volontiers à cette croisade contre les « abus » des plus précaires qu'ils sont convaincus de défendre ainsi leur avenir sous l'aile protectrice de la puissance publique.

(A. Spire, décembre 2013)
Sami, przynależąc do niższych warstw świata pracy, wierzą, że przystępując do krucjaty przeciw popełnianym przez ludzi w najgorszej sytuacji „,nadużyciom”, bronią swojej własnej przyszłości pod ochronnym parasolem opiekuńczego państwa. (A. Dwulit)

L'impression que la pauvreté constitue un moindre tabou dans la version polonaise de l'article est renforcée par l'exemple ci-dessous, où les personnes touchant des allocations donnant un minimum de ressources pour vivre sont nommées directement najbiedniejsi ('les plus pauvres').

(10) Là encore, on retrouve le même argument utilisé par les directeurs des CAF pour obtenir l'assentiment des agents chargés de faire fonctionner cette machine à punir les bénéficiaires de minima sociaux : il n'est question que d'améliorer la qualité du service dans la perspective de mieux garantir l'accès aux prestations de ceux qui sont en droit de les demander.

(A. Spire, décembre 2013)
By uzyskać aprobatę dla maszyny do karania najbiedniejszych, znowu stosuje się ten sam argument: chodzi przecież tylko o poprawę jakości działania służb, których celem jest zapewnienie w przyszłości jak najlepszego dostępu do świadczeń tym, którzy naprawdę mają prawo się ich domagać.

(A. Dwulit)

La notion quartiers défavorisés est concurrencée dans le discours de presse par quartiers populaires, prioritaires, en difficulté, précaires, sensibles, pauvres etc. (Seoane, 2016, p. 275). Le $P R$ explique l'adjectif défavorisé comme suit : «Désavantagé sur le plan économique, social ou culturel». Il mentionne par la suite la notion de milieux défavorisés, synonyme de quart-monde, ainsi que les quartiers les plus défavorisés de la ville, une dénomination des quartiers déshérités et pauvres. Dans la version polonaise de l'éditorial Droitisation, mode d'emploi de S. Halimi, les traductions des notions en question pourraient être considérées comme correspondantes, avec cependant cette objection que les expressions proposées par le traducteur ne sont point complaisantes, au contraire, elles renchérissent l'idée d'exclusion et sont plus stigmatisantes :

(11) Lorsque, le 11 janvier dernier, des millions de Français ont manifesté leur solidarité avec les victimes des attentats de Paris, la mobilisation des classes moyennes a contrasté une fois de plus avec celle, relativement plus modeste, du monde ouvrier et de la jeunesse des quartiers défavorisés.

(S. Halimi, juin 2015)
Kiedy 11 stycznia br. miliony Francuzów manifestowały swoją solidarność z ofiarami zamachów w Paryżu, mobilizacja klasy średniej po raz kolejny kontrastowała ze względnie skromniejszą mobilizacją świata pracy i młodzieży $\mathbf{z}$ dzielnic upośledzonych.

(Z.M. Kowalewski) 
(12) «La désaffection grandissante des milieux populaires pour les partis de gauche, observable dans toutes les démocraties occidentales électives, relève le politiste Patrick Lehingue, n'est sans doute pas sans rapport avec la raréfaction des élus qui, issus des milieux défavorisés, en avaient éprouvé les conditions d'existence ». (S. Halimi, juin 2015)
„Narastające zniechęcenie środowisk ludowych partiami lewicowymi, które daje się zaobserwować we wszystkich zachodnich demokracjach parlamentarnych - stwierdza politolog Patrick Lehingue - niewątpliwie nie pozostaje bez związku z narastającą rzadkością posłów i radnych, którzy wywodzą się ze środowisk upośledzonych i doświadczyli ich warunków bytowych". (Z.M. Kowalewski)

On retrouve dans les articles français les formulations franges fragiles, zones fragiles où l'adjectif fragile renvoie d'une manière édulcorée à des problèmes sociaux liés à la pauvreté, au chômage, à l'exclusion. Il entre dans le paradigme : quartier fragile, situation socio-économique fragile etc. Dans l'exemple cité, l'adjectif fragile qualifiant les zones est le synonymes de sensible, instable, précaire, risquant de se détériorer, alors que dans la version polonaise on parle de strefy podupadte ('zones délabrées / dégradées'). Nous observons donc un décalage métonymique : potentialité (en français) - fait accompli (en polonais).

(13) En France, le FN séduit surtout dans les zones fragiles, où les jeunes sans diplôme sont nombreux et les taux de chômage et de pauvreté très élevés.

(B. Bréville, avril 2017)
We Francji FN uwodzi wyborców przede wszystkim w strefach podupadłych, gdzie wielu jest młodych ludzi bez dyplomów, a stopy bezrobocia i ubóstwa są wysokie.

(Z.M. Kowalewski)

De même, banlieues populaires ou quartiers populaires sont traduits à plusieurs reprises comme biedne dzielnice. Dans le fragment ci-dessous, on peut observer que le ton du texte polonais est plus expressif par rapport à l'original ; d'un côté à cause de l'adjectif biedne ('pauvres') qui décrit les quartiers, et de l'autre parce que l'expression neutre jeune des cités a été traduite avec le substantif argotique et connoté péjorativement blokers qui évoque l'image d'un jeune de grands ensembles habités par des familles pauvres, appartenant à une sous-culture hip-hop ${ }^{5}$ :

(14) Valorisée par l'État à travers des programmes comme le concours «Talents des cités», imaginé en 2002 pour récompenser de jeunes créateurs et entrepreneurs installés dans des quartiers populaires, la figure de l'immigré ou du « jeune des cités qui s'en est sorti » prospère également dans les médias. (H. Belmessous, mars 2017)
W mediach stale obecna jest postać imigranta czy „blokersa”, który zrobił karierę, promowana przez władze poprzez takie programy, jak chociażby istniejący od 2002 r. konkurs ,Talents des cités”, nagradzający młodych twórców i przedsiębiorców działających w biednych dzielnicach.

(A. Dwulit)

\footnotetext{
${ }^{5}$ sjp.pwn.pl, sjp.pl, dobryslownik.pl
} 
Il résulte de cette courte comparaison que le lexème pauvre est évité dans la version française du magazine alors qu'il est employé plus facilement dans les traductions polonaises. On pourrait se demander si c'est uniquement l'effet des sensibilités subjectives des traducteurs ou des différences dans les orientations éditoriales, ou plutôt des divergences dans la doxa des deux sociétés, qui font que la pauvreté est un moindre tabou dans la langue polonaise, ce qui resterait à démontrer sur des corpus plus vastes.

\section{LA NÉGOCIATION DES NOTIONS}

La lecture des articles dans les deux langues fait surgir les traces de l'intervention du traducteur dans l'interprétation de certaines notions. Dans le fragment ci-dessous, on observe une discussion des notions entre l'auteur de l'original, en l'occurrence le Front national, et le traducteur de l'article dans lequel est cité le programme de ce parti. Nous retrouvons ici un débat politique ou sémantique actuel concernant la définition des termes : migrant et réfugié. En effet, dans le fragment cité dans l'article, il est question de migrants clandestins alors que la traductrice rend ce terme avec un terme polonais plus restreint: uchodźcy ('réfugiés'). Un migrant est considéré comme un hypéronyme par rapport au réfugié et il désigne celui «qui participe à une migration, c'est-à-dire à un déplacement de populations qui passent d'un pays dans un autre pour s'y établir » $(P R)$. Nous retrouvons la même relation sémantique entre les notions polonaises imigrant-uchodźca, ce qui n'est pas étonnant car ces termes sont définis par le droit international. On peut observer que le $P R$ de l'année 2015 donnait seulement un sens spécialisé du terme migrant : «travailleur originaire d'une région peu développée, s'expatriant pour des raisons économiques ». Toutefois, la version 2017 de ce dictionnaire ajoute cette acception nouvelle : «Personne qui fuit son pays pour échapper à un conflit armé $\rightarrow$ réfugié ». Bonhomme juge qu' « on peut estimer que son extension [du terme migrant] aux victimes de guerres (Syrie, Afghanistan...) constitue un euphémisme néologique, par élargissement sémantique ou par affectation à un nouveau référent» (Bonhomme, 2016, p. 231). Le choix des mots n'est pas anodin, qu'il s'agisse du français ou du polonais, il reflète l'attitude du scripteur envers les personnes concernées. C'est pourquoi, la réception du texte polonais est différente si l'on traduit migrant par (i)migrant ou uchodźca, car ces deux termes véhiculent d'autres informations sur les conditions de vie des personnes concernées, de même que sur les obligations du pays d'accueil envers elles : 
(15) Le programme du Front national (FN) préconise ainsi : "Restaurer l'équilibre des comptes sociaux en donnant la priorité aux Français : instaurer un délai de carence d'un an de résidence continue en France et de cotisation avant de bénéficier de tous les avantages de la Sécurité sociale ; supprimer l'AME [aide médicale de l'Etat] réservée aux migrants clandestins ; créer un observatoire des droits sociaux des étrangers et de l'usage des conventions bilatérales de soins ».

(A. Spire, décembre 2013)
W poświęconej problemom zdrowia części partyjnego programu Frontu Narodowego czytamy: „Odbudować równowagę finansową, dając priorytet Francuzom [...]; ustanowić okres karencji - osoby korzystające z dobrodziejstw opieki społecznej muszą co najmniej od roku bez przerwy przebywać we Francji i płacić składki; znieść państwową pomoc medyczną [AME] dla nielegalnych uchodźców; stworzyć obserwatorium praw społecznych obcokrajowców i stosowania dwustronnych konwencji dotyczących ochrony zdrowia; walczyć z oszustwami i nadużyciami”.

(A. Dwulit)

L'extrait suivant présente également le programme du F.N. Nous y avons souligné deux expressions. Le terme français expulsion n'est pas rendu en polonais avec le terme administratif wydalenie ('l'action de contraindre quelqu'un à quitter un lieu, renvoyer') qui souligne le caractère légal de l'action (Dubisz, 2003), mais avec le lexème wypędzenie (l'action de forcer quelqu'un à quitter un lieu, chasser') (Dubisz, 2003) lequel comporte le sème /+ force physique/. Ainsi, le traducteur accentue le caractère de violence physique qui peut être liée à la mesure proposée. La distance du traducteur/rédacteur par rapport au programme du parti cité est renforcée par une mise entre guillemets de la formule preferencja narodowa, traduction de préférence nationale. Ceci attire en même temps l'attention du lecteur sur cette expression. «L'hétérogénéité graphique ainsi créée suggère aux lecteurs d'effectuer une réflexion métalinguistique » (Bonhomme, 2005, p. 80).

\begin{tabular}{|l|l|}
\hline (16) Le Pen (Front national, FN) s'engage à suppri- & Marine Le Pen (Front Narodowy, FN) zobowią- \\
mer le droit du sol, à sortir de l'espace Schen- & zuje się, że zniesie ius soli, opuści przestrzeń \\
gen, à instaurer la préférence nationale et & Schengen, zaprowadzi „preferencję narodową” \\
à systématiser les expulsions d'étrangers en & i usystematyzuje wypędzanie cudzoziemców bez \\
situation irrégulière. & papierów. \\
(B. Bréville, avril 2017) & (Z.M. Kowalewski)
\end{tabular}

Le fragment suivant est un exemple contraire, plus rare dans les textes analysés, où le traducteur atténue l'expression de l'original, désignant cette fois-ci une réalité de la culture cible. L'article Le carburant social de la droite polonaise, comme tous les autres articles cités, a été rédigé en français et est paru d'abord dans l'édition française du Monde diplomatique, puis après sa traduction, dans l'édition polonaise. Le parti PiS est nommé à plusieurs reprises dans le texte, il est désigné une fois par une périphrase : ce parti situé à droite de la droite, autrement dit de droite radicale. Dans l'édition polonaise, nous lisons ta prawicowa partia ('ce parti de droite'). L'expression, qui aurait pu être controversée par certains, a donc été modérée. 
(17) Mineur et délégué syndical Solidarność, Kalabis, związany z Solidarnością, też popiera tę M. Kalabis milite aussi pour ce parti situé à la droite de la droite.

(C. Gouverneur, mars 2016)

partię

(A. Dwulit)

À la lecture de ces courts exemples, l'on peut constater que les choix des traducteurs entre des lexèmes présentant des différences subtiles de sens peuvent avoir des conséquences importantes pour le sens du message véhiculé.

\section{CONCLUSION}

Il est vrai qu'une étude sur les traductions de presse qui émanent d'instances moins rapprochées ou même opposées idéologiquement par rapport aux auteurs du texte source, ferait surgir des différences plus accrues reflétant des attitudes polémiques envers l'original. Pourtant, l'analyse des textes proches idéologiquement permet d'observer des divergences qui ne découlent pas d'une interprétation polémique, mais d'autres phénomènes, notamment langagiers. Ainsi, nous avons observé, concernant la guerre, des euphémismes analogues, voire correspondants car il s'agit d'internationalismes calqué sur l'anglais. L'emploi de ces expressions est similaire dans les deux versions, y compris en ce qui concerne une distanciation des auteurs par rapport aux formules citées, ou leur dévoilement. En revanche, cette courte comparaison a aussi permis de constater que la pauvreté constitue un plus grand tabou pour les rédacteurs français qui emploient plusieurs formulations politiquement correctes. Ces menus écarts dans les manières de dénommer les personnes touchées par la pauvreté peuvent laisser supposer des phénomènes langagiers plus généraux qui font que les lexèmes pauvre/pauvreté sont considérés comme stigmatisants et donc évités dans le discours. Certes, plusieurs facteurs influencent l'application de tel ou tel procédé de traduction, que ce soit les systèmes linguistiques, le cotexte ou les paramètres situationnels de la traduction, notamment la culture et les lecteurs cibles, le genre journalistique, la ligne éditoriale, la subjectivité $\mathrm{du}$ traducteur, de même que les tendances des traducteurs à choisir certaines méthodes de traduction observables dans le troisième code. Ces écarts entre l'original et la traduction autorisent à poser la question de savoir à qui ils sont dus : au traducteur, aux rédacteurs, au discours, à la société. Etant donné que le traducteur est immergé dans l'espace et le temps, il reflète la doxa sociale. L'euphémisation sert à une recatégorisation dans le discours public d'un côté, elle exprime la doxa, de l'autre, elle contribue ainsi à établir des systèmes de valeurs qui constituent des points de repères de l'identité sociale, lesquels sont dynamiques et évoluent (Dyoniziak, 2017). 
D'autre part, les choix entre les lexèmes de différences subtiles entraînent des conséquences importantes dans le sens du message. Ainsi, par exemple la réception de la proposition du FN de systématiser les expulsions des étrangers sera différente si l'on traduit expulsion par wydalenie ou wypędzenie. La tendance à atténuer ou à accentuer les connotations péjoratives laissent deviner la position des auteurs de la version polonaise envers les mesures proposées.

L'étude comparative des mêmes textes de presse en deux langues laisse observer les choix interprétatifs des traducteurs qui témoignent de leur subjectivité ou de la ligne éditoriale, mais aussi à travers eux, permet de comparer des traits de discours public dans les deux langues. C'est pourquoi elle devrait être continuée sur des corpus plus larges. 


\section{RÉFÉRENCES BIBLIOGRAPHIQUES}

Baker, M. (1996). " Corpus-based Translation Studies: The Challenges that Lie Ahead ». In H. Somers (réd.), Terminology, LSP and Translation: Studies in Language Engineering, in Honour of Juan C. Sager (pp. 175-186). Amsterdam/Philadelphia : John Benjamins.

Baker, M. (1998). « Réexplorer la langue de la traduction : une approche par corpus ». Meta, 43 (4), 480-485. DOI : 10.7202/001951ar.

Balcerzan, E. (2011). Ttumaczenie jako wojna światów. W kręgu translatoryki i komparatystyki. Poznań : Wydawnictwo Naukowe UAM.

Bonhomme, M. (2005). Pragmatique des figures du discours. Paris : Honoré Champion.

Bonhomme, M. (2012). "La réception de l'euphémisme : entre réussite et échec interactif ». In A. Horak, M. Torre \& M. Bonhomme (réd.), Études pragmatico-discursives sur l'euphémisme. Estudios pragmatico-discursivos sobre el eufemismo (pp. 73-89). Frankfurt am Main : Peter Lang.

Bonhomme, M. (2016). "La néologie euphémique dans la presse suisse romande ». La linguistique, 52, 223-238. DOI : 10.3917/ling.522.0223.

Bonhomme, M., Torre, M. \& Horak, A. (2012). « Présentation ». In A. Horak, M. Torre \& M. Bonhomme (réd.), Études pragmatico-discursives sur l'euphémisme. Estudios pragmatico-discursivos sobre el eufemismo (pp. 7-20). Frankfurt am Main : Peter Lang.

Dąmbska-Prokop, U. (2000). Mata encyklopedia przekładoznawstwa. Częstochowa : Edukator.

Dumarsais, C.C. (1988). Des tropes. Paris : Flammarion.

Dyoniziak, J. (2017). «L'euphémisme face à la construction de l'identité sociale. Le cas du discours médiatique ». In A. Konowska \& A. Bobińska (réd.), Nouveaux tabous linguistiques (pp. 97-112). Łódź : Wydawnictwo Uniwersytetu Łódzkiego.

Frawley, W. (1984). " Prolegomenon to a Theory of Translation ». In W. Frawley (réd.), Translation: Literary, Linguistic, and Philosophical Perspectives (pp. 159-175). London/Toronto : Associated University Presses.

Guidère, M. (2010). Introduction à la traductologie : penser la traduction : hier, aujourd'hui, demain. Bruxelles : De Boeck.

Lavault-Olléon, E. \& Sauron, V. (2009). « Journaliste et traducteur : deux métiers, deux réalités ». ILCEA, 11. Accessible sur le site http://ilcea.revues.org/210.

López Diaz, M. (2012). " Dire pour masquer ou la stratégie de l'euphémisme publicitaire ». In A. Horak, M. Torre \& M. Bonhomme (réd.), Etudes pragmatico-discursives sur l'euphémisme. Estudios pragmatico-discursivos sobre el eufemismo (pp. 193-208). Frankfurt am Main : Peter Lang.

López Diaz, M. (2017). « Lexique et locutions euphémistiques dans le domaine de l'emploi ». In. A. Konowska \& A. Bobińska (réd.), Nouveaux tabous linguistiques (pp. 19-30). Łódź: Wydawnictwo Uniwersytetu Łódzkiego.

Moirand, S. (2007). Les discours de la presse quotidienne. Observer, analyser, comprendre. Paris : Presses Universitaires de France.

Pierret, R. (2013). «Qu'est-ce que la précarité ? ». Socio, 2, 307-330. DOI : 10.4000/socio.511.

Seoane, E. (2016). « Deux néologismes par glissement sémantique : quand l'euphémisme cristallise ». La linguistique, 52, 271-290. DOI : 10.3917/ling.522.0271.

Tomaszkiewicz, T. (2016). "Présence du traducteur dans le processus de traduction spécialisée ». Studia Romanica Posnaniensia, XLIII (1), 93-107. DOI : 10.14746/strop.2016.425.007. 


\section{DICTIONNAIRES}

Dubisz, S. (2003). Uniwersalny stownik języka polskiego. Warszawa : Wydawnictwo Naukowe PWN.

Rey-Debove, J. \& Rey, A. (réd.). (2017). Dictionnaire alphabétique et analogique de la langue française. Paris : Le Robert.

Dobry słownik języka polskiego, https://dobryslownik.pl.

Stownik języka polskiego, https://dobryslownik.pl.

Słownik języka polskiego PWN, https://sjp.pwn.pl. 